\title{
Non-Gaussian statistics of an optical soliton in the presence of amplified spontaneous emission
}

\author{
S. A. Derevyanko* and S. K. Turitsyn \\ Photonics Research Group, Aston University, Birmingham B4 7ET, UK \\ D. A. Yakushev \\ Institute for Radiophysics and Electronics of the National Academy of Sciences of Ukraine, Kharkov 61085, Ukraine \\ Received April 28, 2003

\begin{abstract}
We apply an approach based on the Fokker-Planck equation to study the statistics of optical soliton parameters in the presence of additive noise. This rigorous method not only allows us to reproduce and justify the classical Gordon-Haus formula but also leads to new exact results. () 2003 Optical Society of America

OCIS codes: $060.2330,190.5530$.
\end{abstract}

Since the seminal work by Gordon and $\operatorname{Haus}^{1}$ (see also Ref. 2 for the mathematical theory of the effect), a great deal of attention has been devoted to studying the statistics of the soliton parameters in a long-haul optical communication. Amplified spontaneous emission yields spurious jitter in the soliton parameters, which in turn impairs transmission. The effect is enhanced as the propagation distance increases. For instance, the celebrated Gordon-Haus result for the pure nonlinear Schrödinger equation with lumped amplifiers says that the variance of the time jitter grows proportionally to the cube of the propagation distance.

Most results concerning soliton jitter have been obtained under the assumption that the statistics is Gaussian. Although the results based on this assumption seem to agree rather well with numerics and experiments, from the theoretical view point a more rigorous justification is desirable. Actually, there is no reason to assume that Gaussian statistics holds for large propagation distances, because the system under consideration is essentially nonlinear.

Knowledge of the correct probability density function (PDF) is especially important because such characteristics as bit-error rate depend on the shape of the whole PDF, in particular, on its tails. Large, rare fluctuations in a nonlinear system are typically beyond the area of applicability of the usual Gaussian statistics. ${ }^{3-10}$ Therefore knowledge of the whole PDF, including tails, is absolutely crucial for a correct estimate of the bit-error rate. And, as we show below, these tails are not Gaussian even for small propagation distances, when the bulk of the PDF can still be considered as such.

In this Letter we study soliton statistics, rigorously deriving the Fokker-Planck equation governing the PDF for the four soliton parameters. As the principal example we examine the nonlinear Schrödinger equation with additive white Gaussian noise. First we show that the Gaussian statistics can be justified for short enough propagation distances (apart from the tails). For large distances the Gaussian approximation breaks down, and we illustrate this by calculating explicitly the PDF of the soliton amplitude.

In the presence of amplified spontaneous emission noise the propagation of solitons in optical fibers is described by the perturbed nonlinear Schrödinger equation:

$$
\frac{\partial q}{\partial z}=\frac{i}{2} \frac{\partial^{2} q}{\partial \tau^{2}}+i|q|^{2} q+n(\tau, z) .
$$

Here the normalization and units are taken from Ref. 10. In Eq. (1), $q$ is an envelope of the electric field, $\tau$ and $z$ are the time and propagation distance, respectively, and $n$ is the additive white Gaussian noise term. The amplified spontaneous emission is modeled with the following statistics:

$$
\begin{aligned}
\langle n(\tau, z)\rangle & =\left\langle n(\tau, z) n\left(\tau^{\prime}, z^{\prime}\right)\right\rangle=0, \\
\left\langle n(\tau, z) n^{*}\left(\tau^{\prime}, z^{\prime}\right)\right\rangle & =D \delta\left(z-z^{\prime}\right) \delta\left(\tau-\tau^{\prime}\right) .
\end{aligned}
$$

Without the noise term $n(\tau, z)=0$, the fundamental soliton solution of Eq. (1) is

$q_{0}(\tau, z)=A(z) \operatorname{sech}\{A(z)[\tau-T(z)]\} \exp [-i \Omega(z) \tau+i \phi(z)]$,

where

$$
\begin{aligned}
A(z) & =A_{0}, \\
\Omega(z) & =\Omega_{0}, \\
\phi(z) & =\phi_{0}+\frac{1}{2}\left({A_{0}}^{2}-\Omega_{0}{ }^{2}\right) z, \\
T(z) & =T_{0}-\Omega_{0} z
\end{aligned}
$$

where the constants $A_{0}, \Omega_{0}, \phi_{0}$, and $T_{0}$ are the values of $A(z), \Omega(z), \phi(z)$, and $T(z)$, respectively, at $z=0$. In what follows, without loss of generality, we assume that $T_{0}=\Omega_{0}=\phi_{0}=0$.

Since the noise intensity $D$ is always small, one can apply adiabatic soliton perturbation theory (see, for instance, Refs. 8 and 10-12). In the first order of perturbation theory, the slow changes of the soliton parameters are decoupled from the background excitations. Equations (5)-(8) acquire noisy perturbation terms ${ }^{10}$ : 


$$
\begin{aligned}
\frac{\mathrm{d} A}{\mathrm{~d} z}= & \operatorname{Re} \int \mathrm{d} \tau g_{A}{ }^{*}(\tau) n(\tau, z), \\
\frac{\mathrm{d} \phi}{\mathrm{d} z}= & \frac{1}{2}\left(A^{2}-\Omega^{2}\right)+T \frac{\mathrm{d} \Omega(z)}{\mathrm{d} z} \\
& +\operatorname{Re} \int \mathrm{d} \tau g_{\phi}{ }^{*}(\tau, z) n(\tau, z), \\
\frac{\mathrm{d} \Omega}{\mathrm{d} z}= & \operatorname{Re} \int \mathrm{d} \tau g_{\Omega}{ }^{*}(\tau, z) n(\tau, z), \\
\frac{\mathrm{d} T}{\mathrm{~d} z}= & -\Omega+\operatorname{Re} \int \mathrm{d} \tau g_{T}{ }^{*}(\tau, z) n(\tau, z),
\end{aligned}
$$

where the noise terms are projections of the additive white Gaussian noise $n$ onto four perturbation functions $g_{i}$ :

$$
\begin{aligned}
g_{A}(\tau, z)= & q_{0}(\tau, z, A, \phi, \Omega, T), \\
g_{\phi}(\tau, z)= & \frac{i}{A}\{1-A(\tau-T) \tanh [A(\tau-T)]\} \\
& \times q_{0}(\tau, z, A, \phi, \Omega, T), \\
g_{\Omega}(\tau, z)= & -i \tanh [A(\tau-T)] q_{0}(\tau, z, A, \phi, \Omega, T), \\
g_{T}(\tau, z)= & \frac{\tau-T}{A} q_{0}(\tau, z, A, \phi, \Omega, T) .
\end{aligned}
$$

We emphasize that parameters $A, \phi, \Omega$, and $T$ on the right-hand side of Eqs. (13) -(16) are the current parameters of the soliton that themselves depend on the noise. Therefore Eqs. (9)-(12) form a set of Langevin equations with multiplicative noise, since the functions $g_{i}$ on the right-hand side depend on the random soliton parameters themselves. Note that, if we neglect the fact that functions $g_{i}$ depend on the current values of the parameters and substitute $g_{i}\left(\tau, z, A_{0}, \phi_{0}, \Omega_{0}, T_{0}\right)$ for $g_{i}(\tau, z, A, \phi, \Omega, T)$, we arrive at the Langevin equations with additive Gaussian noises. The statistics then is obviously Gaussian and reproduces the classical Gordon-Haus result for the variances. However, this procedure is not justified for large $z$, and the exact system (9)-(12) should be analyzed instead. For such a system it is possible to derive the Fokker-Planck equation for joint distribution function $P(A, \phi, \Omega, T \mid z)$. Using the standard Stratonovich procedure (details will be published elsewhere), we obtain

$$
\begin{aligned}
\frac{\partial P}{\partial z}= & -\frac{1}{2}\left(A^{2}-\Omega^{2}\right) \frac{\partial P}{\partial \phi}+\Omega \frac{\partial P}{\partial T} \\
& +D\left[\frac{1}{6} A T^{2}+\frac{1}{12 A}\left(2+\frac{\pi^{2}}{6}\right)\right] \frac{\partial^{2} P}{\partial \phi^{2}} \\
& +\frac{D}{3} A T \frac{\partial^{2} P}{\partial \phi \partial \Omega}+\frac{D}{2} A \frac{\partial^{2} P}{\partial A^{2}} \\
& +\frac{D}{6} A \frac{\partial^{2} P}{\partial \Omega^{2}}+\frac{D \pi^{2}}{24 A^{3}} \frac{\partial^{2} P}{\partial T^{2}}
\end{aligned}
$$

Equation (17) should be provided with the initial condition, which is $P(A, \phi, \Omega, T \mid 0)=$ $\delta\left(A-A_{0}\right) \delta(\phi) \delta(\Omega) \delta(T)$. The distribution function should decrease rapidly for $|\Omega| \rightarrow \infty,|T| \rightarrow \infty$. The PDF is also a periodic function in phase $\phi$.

The boundary conditions related to the (positive) amplitude $A$ are as follows: The PDF tends to zero as $A \rightarrow \infty$, and the probability flux through the boundary $A=0$ is equal to zero. For Eq. (17) it implies that $P(0, \phi, \Omega, T \mid z)=0$. We must point out, however, that in the limit of ultrasmall amplitudes, $A \rightarrow 0$, the assumption of the smallness of the noise breaks down and the model defined by Eqs. (9)-(12) in this limit gives only extrapolation of soliton statistics. This must be taken into account when one is applying Eq. (17) to problems for which low-amplitude statistics is important, e.g., the problem of creation of a soliton from noise.

Equation (17) can be rewritten in the canonical form for the multidimensional Fokker-Planck equation:

$$
\frac{\partial P}{\partial z}=-\frac{\partial}{\partial Q_{i}}\left[D_{i}(Q) P\right]+\frac{\partial^{2}}{\partial Q_{i} \partial Q_{j}}\left[D_{i j}(Q) P\right],
$$

where $D_{i}$ are the components of the drift vector and $D_{i j}$ are the elements of the diffusion matrix. Let us first consider the case when propagation distance $z$ is small. Then, because of the initial condition the PDF still displays a sharp peak, while the coefficients $D_{i j}$ are fairly smooth functions of their arguments. Therefore we can substitute the diffusion coefficients for their initial values, $D_{i j}(Q)=D_{i j}\left(Q_{0}\right)$. This assumption does not provide us with the correct tails, since we have expanded the diffusion and drift coefficients near the head of the PDF, but it works rather well when we consider integral characteristics of the distribution such as, for instance, variances.

Under this assumption, Eq. (17) is substantially simplified. In particular, we can integrate off amplitude and phase, using the boundary conditions and arrive at the following equation for the marginal timingfrequency PDF:

$$
\begin{aligned}
\frac{\partial P(\Omega, T)}{\partial z}= & -\frac{\partial}{\partial T}(-\Omega P)+\frac{\partial^{2}}{\partial \Omega^{2}}\left(\frac{D}{6} A_{0} P\right) \\
& +\frac{\partial^{2}}{\partial T^{2}}\left(\frac{D \pi^{2}}{24 A_{0}{ }^{3}} P\right) .
\end{aligned}
$$

Equation (19) has a standard form of Fokker-Planck equation for the Ornstein-Ulenbeck process. ${ }^{13}$ Its solution is well known and is Gaussian. We may now calculate the covariance matrix of this Gaussian distribution. One can verify that the diagonal components of the covariational matrix are found to be exactly the same as in the classical Gordon-Haus effect. So we have obtained classical results as a near limit of small propagation distances.

For large propagation distances the whole solution of Eq. (17) is clearly non-Gaussian. We illustrate this by calculating the exact form of the joint PDF for soliton frequency and amplitude. Integration of the 
general Fokker-Planck equation (17) over phase $\phi$ and $T$ yields the autonomous equation for $P(\Omega, A \mid z)$ :

$$
\frac{\partial P}{\partial z}=\frac{D}{2} A \frac{\partial^{2} P}{\partial A^{2}}+\frac{D}{6} A \frac{\partial^{2} P}{\partial \Omega^{2}} .
$$

Integration of this equation over frequency $\Omega$ yields an autonomous equation for $P(A)$ only. A Fokker-Planck equation for the soliton amplitude in the perturbed nonlinear Schrödinger system was reported in Ref. 14. However, the Langevin system considered in Ref. 14 was different from the system considered here. In particular, in Ref. 14, the noise terms in the equations for the soliton phase [Eq. (10)] and position [Eq. (12)] have been neglected, resulting in a different equation for $P(A)$. We stress, however, that these terms are highly relevant, since they contribute to the drift coefficient of the soliton amplitude. These terms must be kept to obtain correct expressions for both joint $P(A, \phi, \Omega, T)$ and marginal $P(A)$ PDFs.

Applying the Fourier transform in $\Omega$ and the Laplace transform in $A$, we arrive at an equation that can be solved with the method of characteristics. Omitting the details, we present the joint PDF in the form

$$
P(\Omega, A \mid z)=\frac{1}{2 \pi} \int_{-\infty}^{\infty} P_{k}\left(A, z^{\prime}\right) \exp (-i k \Omega) \mathrm{d} k,
$$

where

$$
\begin{aligned}
P_{k}\left(A \mid z^{\prime}\right)= & \left(\frac{A}{A_{0}}\right)^{1 / 2} \frac{\alpha_{k}}{\sinh \left(\alpha_{k} z^{\prime}\right)} \exp \left[-\alpha_{k}\left(A+A_{0}\right)\right. \\
& \left.\times \operatorname{coth}\left(\alpha_{k} z^{\prime}\right)\right] I_{1}\left[\frac{2 \alpha_{k} \sqrt{A A_{0}}}{\sinh \left(\alpha_{k} z^{\prime}\right)}\right],
\end{aligned}
$$

$I_{1}$ is the modified Bessel function, $z^{\prime}=(D / 2) z$, and $\alpha_{k}=k / \sqrt{3}$. Integrating Eq. (22) over frequency, we obtain the PDF for the soliton amplitude jitter $P(A)=$ $\int P(\Omega, A \mid z) \mathrm{d} \Omega$ :

$$
P\left(A \mid z^{\prime}\right)=\frac{1}{z^{\prime}}\left(\frac{A}{A_{0}}\right)^{1 / 2} \exp \left(-\frac{A+A_{0}}{z^{\prime}}\right) I_{1}\left(\frac{2 \sqrt{A A_{0}}}{z^{\prime}}\right) .
$$

Equation (23) represents an explicit form of the non-Gaussian PDF $P(A)$. Using an asymptotic expansion of the Bessel function, one can verify that it has an exponential tail, as calculated in Ref. 8:

$$
P(A) \sim\left(\frac{A}{A_{0}}\right)^{1 / 2} \exp \left[-\frac{\left(\sqrt{A}-\sqrt{A_{0}}\right)^{2}}{z^{\prime}}\right], \quad A \rightarrow \infty .
$$

By use of the exact PDF (23) it is easy to compute higher-order momenta $\left\langle A^{n}\right\rangle=z^{\prime n} n ! L_{n}{ }^{1}\left(-A_{0} / z^{\prime}\right)$, where $L_{n}{ }^{1}$ are the generalized Laguerre functions. In particular, $\langle A\rangle=A_{0}+D z$, which represents a systematic increase of the soliton amplitude due to noise, as was pointed out in Ref. 8.

We can now justify the classical result for the Gordon-Haus jitter (see, for instance, Ref. 10) that has been obtained formally under the assumption of
Gaussian statistics. The significant deviations from the classical formulas as a result of non-Gaussian corrections occur for large enough distances $D z \gg 1$ (provided that $A_{0} \sim 1$ ). That this is so means that beyond the critical distance, $z_{\mathrm{cr}} \sim D^{-1}$, the classical formulas for the variances of soliton jitters become inapplicable. However, one must also take into account another important scale, $z_{\mathrm{rad}}$, the distance at which the continuum radiation becomes coupled to the soliton degrees of freedom and the adiabatic perturbations theory that was used to derive Eqs. (9)-(12) needs modification. The continuum radiation becomes important at distances $z_{\text {rad }} \sim D^{-1 / 2}<<z_{\text {cr }}$ (see Ref. 8). this means that within the region of applicability of the model the classical results for the soliton jitter require only small corrections. Note, however, the important result given by Eqs. (21) - (24), which assert that the tails of the PDFs are always non-Gaussian even for an arbitrary small propagation distance. Therefore, one has to account for the non-Gaussian shape of the PDF while calculating quantities such as the bit-error rate.

In summary, a Fokker-Planck equation has been derived that can be used to study the nonGaussian statistics of soliton parameters. The classical formulas for soliton jitters are recovered in the limit of small propagation distances $z$. We have shown that the tails of the PDF are always non-Gaussian and illustrated this by calculating explicitly the marginal PDF for the soliton amplitude and frequency.

S. Derevyanko (e-mail, s.derevyanko@aston.ac.uk) acknowledges receiving Intas Young Scientist Fellowship YS 2002-165. This work was also supported by the Leverhulme Trust Project F/00250/B (S. Derevyanko and S. K. Turitsyn).

*Also with the Institute for Radiophysics and Electronics of the National Academy of Sciences of Ukraine, Kharkov 61085, Ukraine.

\section{References}

1. J. P. Gordon and H. A. Haus, Opt. Lett. 11, 665 (1986).

2. J. Elgin, Phys. Lett. A 110, 441 (1985).

3. T. Georges, Opt. Commun. 123, 617 (1996).

4. C. R. Menyuk, Opt. Lett. 20, 285 (1995).

5. F. Kh. Abdullaev, S. A. Darmanyan, and F. Lederer, Opt. Commun. 126, 89 (1996).

6. J. P. Gordon and L. F. Mollenauer, Opt. Lett. 15, 1351 (1990).

7. C. J. McKinstrie and C. Xie, IEEE J. Sel. Top. Quantum Electron. 8, 616 (2002).

8. G. Falkovich, I. Kolokolov, V. Lebedev, and S. Turitsyn, Phys. Rev. E 63, 025601(R) (2001).

9. G. Falkovich, M. G. Stepanov, and S. K. Turitsyn, Phys. Rev. E 64, 067602 (2001).

10. E. Iannone, F. Matera, A. Mecozzi, and M. Settembre, Nonlinear Optical Communication Networks (Wiley, New York, 1998).

11. D. J. Kaup, Phys. Rev. A 42, 5689 (1990).

12. H. A. Haus and Y. Lai, J. Opt. Soc. Am. B 7, 386 (1990).

13. H. Risken, The Fokker-Planck Equation (Springer, New York, 1996).

14. B. A. Malomed and N. Flytzanis, Phys. Rev. E 48, 5 (1993). 